\section{The controversy of using PGA to define remission in $\mathrm{RA}$}

Ricardo J. O. Ferreira, Cátia Duarte, Mwidimi Ndosi, Maarten de Wit, Laure Gossec and J. A. P. da Silva

We read with interest the commentary by van Tuyl and Boers (van Tuyl, L. H. D. \& Boers, M. Remission - keeping the patient experience front and centre. Nat. Rev. Rheumatol. 13, 573-574 (2017)) ${ }^{1}$ referring to our paper on the role of patient global assessment (PGA) in the definition of remission in rheumatoid arthritis (RA) ${ }^{2}$. However, we cannot agree with their interpretation that by suggesting to remove the PGA from the ACR/EULAR remission definition we are "calling for a paradigm change that limits the responsibility of the rheumatologist to prescribing immunosuppressive therapy," or that our proposal is "taking away the incentive to improve RA care by removing the patient's perspective from the remission criteria."

Nothing could be further from the interpretation we made of our own data and from our proposals. What we actually proposed is that the management of RA should be guided by two separate targets: a measure of inflammatory activity (physician's perspective) and a measure of disease impact (patient's perspective).

We advocate that $3 \mathrm{v}$-remission (defined as swollen and tender 28 -joint counts and C-reactive protein in $\mathrm{mg} / \mathrm{dl}$ all $\leq 1$ ) is the most appropriate target for immunosuppressive therapy given that PGA has been shown to have no more than a weak correlation with disease activity, and is at least as much linked to personality and emotional aspects, which are not amenable to change by immunosuppressive therapy.

Achieving 3v-remission is a decisive step towards achieving good patient outcomes but does not guarantee the total abrogation of disease impact. In fact, the percentage of patients with RA who are missing remission solely because of a high PGA score is greater than the percentage who achieve full remission ${ }^{2,3}$. To further assist such patients, physicians ought to consider adjuvant interventions instead of reinforced immunosuppression.

For these reasons, a measure of disease impact should be part of the recommended treatment targets in RA management. This measure should be examined separately from inflammatory activity and include more analytical measures than PGA, in order to guide efforts to alleviate impact beyond what is achieved through control of inflammation. We suggest that the Rheumatoid Arthritis Impact of Disease (RAID) score, using its seven domains as separate items, is ideally suited for this purpose. The RAID score was developed in close cooperation with patients from various countries ${ }^{4}$.

Our views were summarized in the abstract: "PGA mainly reflects fatigue, pain, function, and psychological domains, which are inadequate to define the target for immunosuppressive therapy. This consideration suggests that clinical practice should be guided by two separate remission targets: inflammation (3v-remission) and disease impact." 2

In summary, we do not propose to "limit the responsibility of the rheumatologist to prescribing immunosuppressive therapy" ${ }^{1}$, but rather we want to highlight the rheumatologist's and multidisciplinary team's responsibility to assess and manage disease impact. The appropriateness of these proposals will be further scrutinized by clarifying whether high PGA in patients otherwise in remission is associated with subclinical inflammation and whether full remission is a better predictor than $3 \mathrm{v}$-remission (without PGA) of a long-term good radiological outcome $^{5}$. Both investigations are underway.
Ricardo J. O. Ferreira ${ }^{1,2 *}$, Cátia Duarte ${ }^{1,3}$, Mwidimi Ndosi $^{4}$, Maarten de Wit ${ }^{5,6}$, Laure Gossec ${ }^{7,8}$ and J. A. P. da Silva ${ }^{1,3}$

${ }^{1}$ Rheumatology Department, Centro Hospitalar e Universitário de Coimbra, Coimbra, Portugal.

${ }^{2}$ Health Sciences Research Unit: Nursing (UICiSA:E), Coimbra, Portugal.

${ }^{3}$ Clínica Universitária de Reumatologia, Faculty of Medicine, University of Coimbra, Coimbra, Portugal.

${ }^{4}$ Department of Nursing and Midwifery, Faculty of Health and Applied Sciences, University of the West of England, Bristol, UK.

${ }^{5}$ Patient research partner, EULAR standing committee of People with Arthritis/Rheumatism in Europe (PARE), Zurich, Switzerland.

${ }^{6}$ Department of Medical Humanities, VU University Medical Centre, Amsterdam, Netherlands.

${ }^{7}$ Sorbonne Universités, UPMC Univ. Paris 06, Institut Pierre Louis d'Epidémiologie et de Santé Publique, GRC-UPMC 08 (EEMOIS), Paris, France.

${ }^{8}$ Rheumatology Department, AP-HP, Pitié-Salpetrière Hospital, Paris, France

*e-mail: rferreira@reumahuc.org doi:10.1038/nrrheum.2018.35 Published online 21 Mar 2018

1. van Tuyl, L. H. D. \& Boers, M. Remission - keeping the patient experience front and centre. Nat. Rev. Rheumatol. 13, 573-574 (2017).

2. Ferreira, R. J. O. et al. Suppressing inflammation in rheumatoid arthritis: does patient global assessment blur the target? A practice-based call for a paradigm change. Arthritis Care Res. https://doi.org/10.1002 acr.23284 (2017).

3. Ferreira, R. J. O. et al. Drivers of patient global assessment in patients with rheumatoid arthritis who are close to remission: an analysis of 1588 patients. Rheumatology. (Oxford). 56, 1573-1578 (2017).

4. Gossec, L. et al. Finalisation and validation of the rheumatoid arthritis impact of disease score, a patient-derived composite measure of impact of rheumatoid arthritis: a EULAR initiative. Ann. Rheum. Dis. 70, 935-942 (2011).

5. Ferreira, R. J. O. et al. The impact of patient global assessment in the definition of remission as a predictor of long-term radiographic damage in patients with rheumatoid arthritis: protocol for an individual patient data meta-analysis. Acta Rheumatol. Port. http://www. actareumatologica.pt/ article_abstract.php?id =1197 (2018).

Competing interests

The authors declare no competing interests.

\section{Author contributions}

R.J.O.F. and J.A.P.S researched data for the article and wrote the article. R.J.O.F., C.D., M.N., L.X., M.W., L.G. and J.A.P.S. made substantial contributions to discussion of its content and reviewed and/or edited the manuscript before submission. 